\title{
Highly Emissive Self-assembled Organic Nanoparticles having Dual Color Capacity for Targeted Immunofluorescence Labeling**
}

\author{
By Hyong-Jun Kim, Jiseok Lee, Tae-Hoon Kim, Taek Seung Lee, and Jinsang Kim*
}

Immunofluorescence labeling is a very important analytical technique for probing the structure of living cells. Such labeling is conventionally carried out by using fluorescent organic dyes. However, some drawbacks including a limited range of light emission and particularly poor photo-stability ${ }^{[1]}$ have limited the usage of organic dyes. After the introduction of semiconducting quantum dots ${ }^{[2,3]}$ many research groups have investigated inorganic quantum $\operatorname{dots}^{[4]}$ for immunofluorescence labeling due to their high quantum yield, ${ }^{[5]}$ high molar extinction coefficients, broad absorption with narrow light emission, and good photo physical and chemical stability. ${ }^{[6-9]}$ Despite the promising properties of semiconducting quantum dots for immunofluorescence labeling, cyto-toxicity is a critical problem in any live-cell or animal experiments. ${ }^{[10,11]}$ Alternative choices are dye-loaded latex particles and dye-doped silica colloids having improved photostability compared to conventional dye molecules. However, dye-loaded beads also have a critical limit of brightness due to self-quenching when high density of dyes present at the nanoparticle surface. In this context, developing highly emissive, biocompatible, and chemically readily modifiable luminescent materials is strongly desired.

[*] Prof. J. Kim, Dr. H.-J. Kim, ${ }^{[+]}$T.-H. Kim

Department of Materials Science and Engineering

University of Michigan

Ann Arbor, MI 48109 (USA)

E-mail: jinsang@umich.edu

Prof. J. Kim

Chemical Engineering and Biomedical Engineering

University of Michigan

Ann Arbor, MI 48109 (USA)

Prof. J. Kim, J. Lee

Macromolecular Science and Engineering

University of Michigan

Ann Arbor, MI 48109 (USA)

T.-H. Kim, Prof. T. S. Lee

Department of Advanced Organic Materials and Textile System

Engineering Chungnam National University

Daejon, 305-764 (Korea)

[+] Current address: OLED center, School of Materials Science and Engineering, Seoul National University, Seoul, 151-744, Korea.

[**] H.-J.K. and J.L. contributed equally to this work. This research was supported by the National Science Foundation (BES 0428010), NSF CAREER Award (DMR 0644864), and the Center for Chemical Genomics-Thermo Fisher Grant. T.-H.K. acknowledges Korea Science and Engineering Foundation (KOSEF) for the exchange student support. Supporting Information is available online from Wiley InterScience or from the authors.
Recently, a few papers have been published reporting conjugated organic small molecules that showed higher quantum yield in the solid state than in solution. ${ }^{[12-16]}$ In other words, self-quenching does not exist in these unique molecules. It has opened a new possibility to develop non-toxic organic nanoparticles with enhanced emission for various applications including immunofluorescence labeling. The reason for the lower quantum yield in solution of some of these molecules is believed that the conjugated backbone of the molecules is significantly twisted by steric hindrance ${ }^{[13]}$ and the radiative decay pathway of the resulting twisted chromophores ${ }^{[17]}$ is generally suppressive. Therefore, planarization of the twisted conjugated backbone upon aggregation in the solid state accordingly enhances the emissive property of the molecules. On the other hand, another class of molecules that has a larger quantum yield in the solid state has a different reason for the enhanced emissive property. These molecules in solution have a low quantum yield because they dissipate energy for molecular rotation. Therefore, they have even $20 \%$ higher quantum yield in the solid state because the molecular rotation is hindered by adjacent molecules in the solid state. ${ }^{[12-17]}$

We have developed highly emissive organic nanoparticles by using colloidal self-assembly of a hydroxyphenyl-benzoxazole (HBO) derivative and diacetylene monomers. Various heterocyclic molecules including $\mathrm{HBO}$ have been investigated by many research groups due to their chemical and thermal stability as well as their high electron mobility. HBO has a high extinction coefficient and excellent stability in the UV range and has shown potential as a UV stabilizer. HBO is also known to undergo excited state intramolecular proton transfer (ESIPT) upon photo-excitation as illustrated in Figure 1A.

We synthesized 1,4-di(3-(benzoxazol-2-yl)-4-hydroxyphenyl)2,5-dihexyloxybenzene (DBO) as shown in Figure 1B. The absorption and photoluminescence spectra of DBO are shown in Figure 2C. Dilute solution of DBO in tetrahydrofuran (THF) has absorption $\lambda_{\max }$ at $335 \mathrm{~nm}$ assigned to syn-enol and emits at $518 \mathrm{~nm}$, showing a large Stokes shift due to ESIPT. A nanoparticle dispersion of DBO was prepared by adding THF solution of DBO to water to induce aggregate formation. The prepared nanoparticles in the mixed solution of THF: $\mathrm{H}_{2} \mathrm{O}(1: 9 \mathrm{v} / \mathrm{v})$ show a broad aggregation band above $400 \mathrm{~nm}$ due to Mie scattering (Fig. $2 C) .{ }^{[18]}$ Interestingly their fluorescence quantum yield $\left(\Phi_{\mathrm{F}}\right)$ was $10 \%$, which is more than 3 times larger than the quantum yield of the THF solution (3\%). The enhanced fluorescence emission intensity in the aggregates is believed to originate from a more planar structure induced by J-type aggregation through 
A)

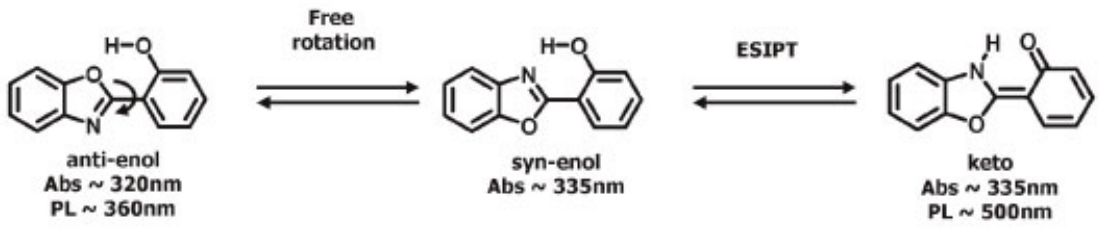

B)
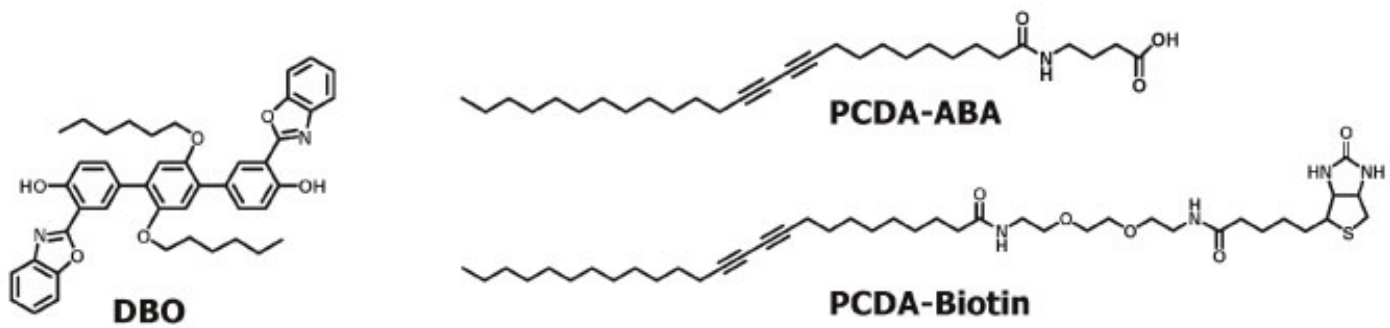

C)
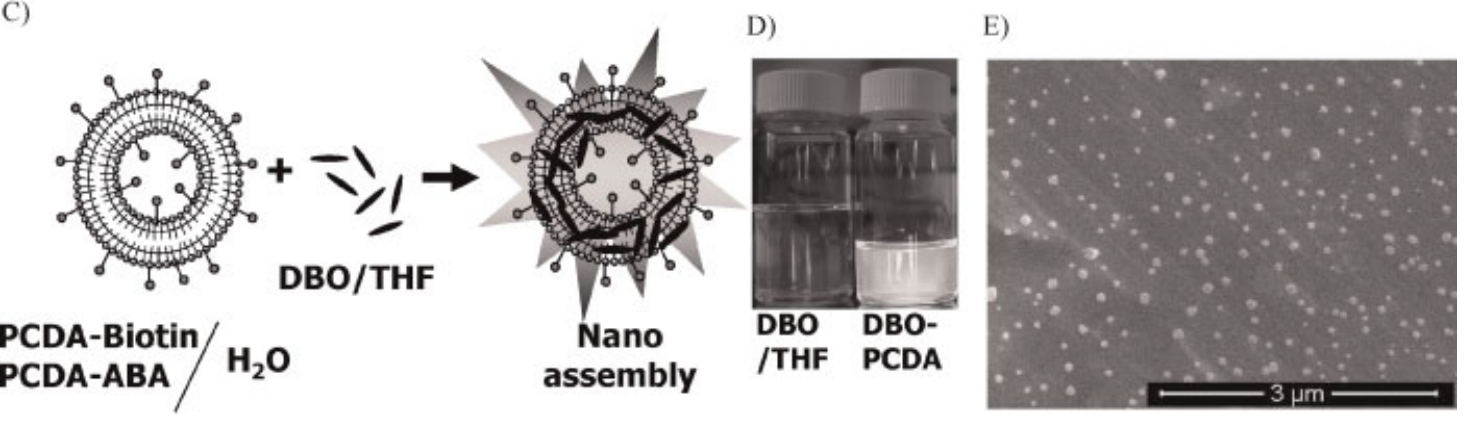

Figure 1. A) Rotamer structures (anti-enol and syn-enol) of $\mathrm{HBO}$ and its excited state keto formation. The given wavelengths are their absorption and emission $\lambda_{\max }$. B) Molecular structures of DBO, PCDA-ABA, and PCDA-Biotin. C) Schematic representation of nanoparticle assembly of DBO in a PCDA vesicle. D) A photograph of DBO in THF solution and DBO-PCDA nanoparticles under $365 \mathrm{~nm}$ UV light. E) Scanning electron microscopy image of the DBOPCDA nanoparticle.

intermolecular hydrogen bonding evidenced by the absorption red shift by $40 \mathrm{~nm}$ from $335 \mathrm{~nm}$ (Fig. 2C).

To reduce the Mie scattering and prepare DBO nanoparticles having a well-defined size, we investigated amphiphilic surfactant molecules in order to direct the self-assembly of DBO. We anticipated that surfactant molecules would disperse DBO aggregates evenly by passivating the surface of the aggregates. Several amphiphilic molecules such as phospholipids, ionic surfactants, and diacetylenes were used to disperse and assemble DBO or random aggregates of DBO into more defined nanoparticle structures. We found that all the amphiphilic molecules served well as a dispersion agent. In this report, we present the results obtained by using a unique combination of diacetylene molecules as a dispersion agent. ${ }^{[19]} 7: 3$ mole ratio of 10,12-pentacosadiynoic acid-aminobutyric acid (PCDAABA) and 10,12-pentacosadiynoic acid-2,2'-(ethylenedioxy)bis(ethylamide)-biotin (PCDA-Biotin) were mixed in HEPES buffer $(0.5 \mathrm{mM})$ and then sonicated to form PCDA vesicles. $0.1 \mathrm{ml}$ of $1 \mathrm{mM}$ DBO in THF solution was then added into the 10 $\mathrm{ml}$ of PCDA vesicle solution and the mixture solution was sonicated for $10 \mathrm{~min}$ to form well-defined $(80 \pm 10) \mathrm{nm}$ of DBO nanoparticles having PCDA passivation layer as illustrated in Figure $1 \mathrm{C}$. The resulting suspension has a 1:50 mole ratio of
DBO to PCDA and the SEM image of the self-assembled DBO-PCDA nanoparticles is shown in Figure 1E. Note the significantly enhanced fluorescence emission intensity of the self-assembled DBO-PCDA nanoparticles in Figure 1D and 2C. The quantum yield of the self-assembled DBO-PCDA nanoparticles was $38 \%$, which is almost 13 times larger than that of DBO in THF solution. It is also interesting to note that the DBO-PCDA nanoparticles have strong anti-enol absorption at $320 \mathrm{~nm}$. Considering the fact that only the syn-enol form can undergo ESIPT and the molecular rotation from antienol to syn-enol is suppressed in the solid state, observing the strong emission at $518 \mathrm{~nm}$ with ca. $200 \mathrm{~nm}$ of Stoke shift was puzzling. ${ }^{[20]}$ We hypothesize that DBO in the DBO-PCDA nanoparticles forms J-type aggregation through intermolecular hydrogen bonding as illustrated in Figure 2A. The planarized conjugated backbone of DBO and the restricted molecular rotation in the $\mathrm{J}$-aggregates are then reasonably believed as the origin of the largely enhanced $38 \%$ quantum yield. ${ }^{[13,17]}$ First, as theoretical energy calculation studies and experimental data showed that twisted conjugated organic molecules generally have lower quantum yield, ${ }^{[12-16]}$ it is reasonable that the planarized conjugated backbone of DBO in the self-assembled J-aggregates has a larger quantum yield compared to DBO in 
A)

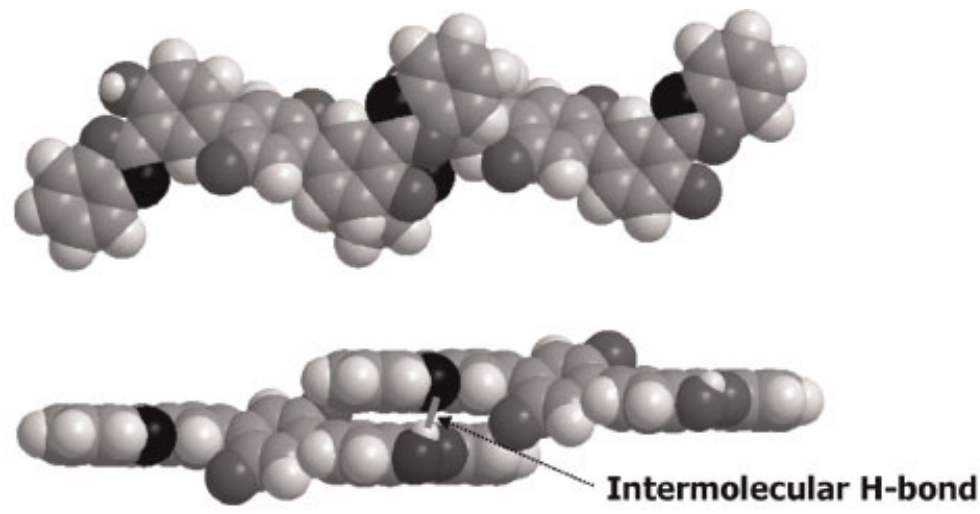

B)

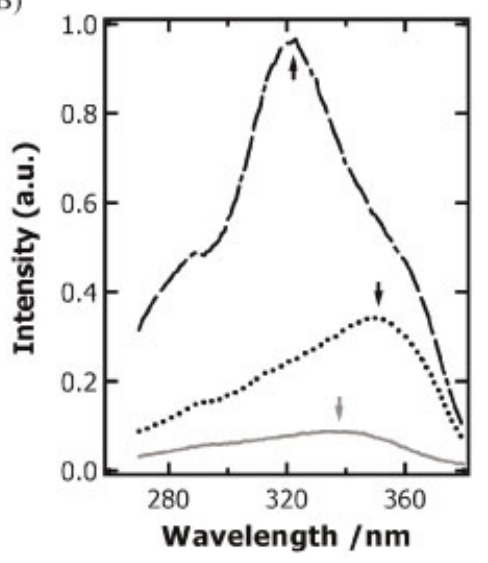

C)

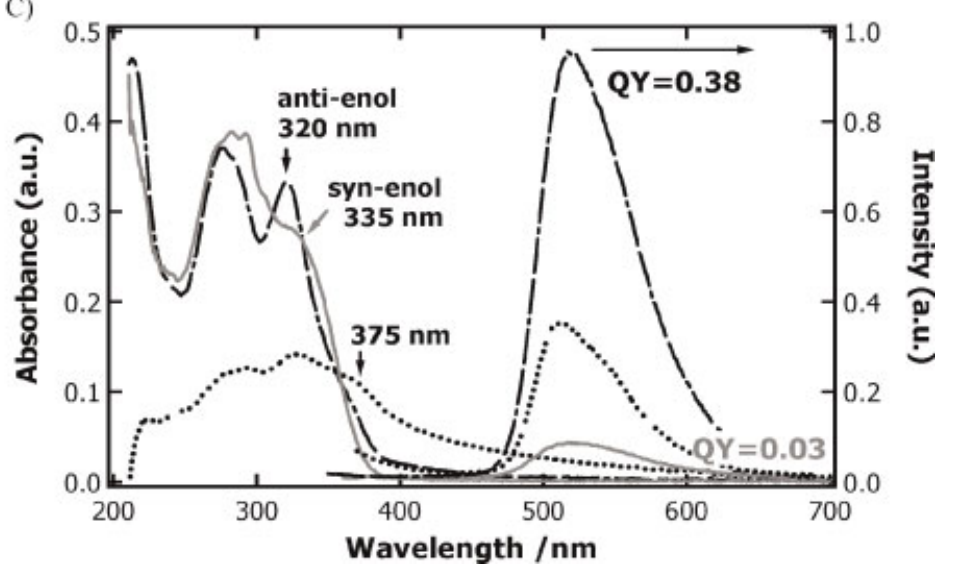

Figure 2. A) Geometry optimized molecular structure of DBO produced by using Materials Studio 4.1 with DFT (density functional theory) with BLYP parameter. Two DBOs are organized to show intermolecular hydrogen bonding between benzoxazole units. (B) Excitation spectra of DBO in THF solution (solid line), dispersion in deionized water (dotted line), and dispersion in diacetylene vesicle (broken line) for the $520 \mathrm{~nm}$ emission of Figure 2C. C) UV/Vis absorption and photoluminescence spectra of DBO in THF solution (solid lines), in THF: $\mathrm{H}_{2} \mathrm{O}(1: 9 \mathrm{v} / \mathrm{v})$ (dotted lines), and in the diacetylene vesicle solution (broken lines). The concentration of the DBO solution and dispersions was $10 \mu \mathrm{M}$.

solution. Another supporting experimental evidence for our hypothesis is the fact that we obtained much longer life-time from the self-assembled DBO-PCDA nanoparticles than the DBO in THF solution. The lifetime of the $518 \mathrm{~nm}$ emission band when excited at $380 \mathrm{~nm}$ was 0.42 and $6.26 \mathrm{~ns}$ for the DBO solution in THF and the DBO-PCDA nanoparticles, respectively. The life-time of the $518 \mathrm{~nm}$ emission band when the DBO-PCDA nanoparticles were excited at $310 \mathrm{~nm}$ (anti-enol) was $6.41 \mathrm{~ns}$ which is similar to the $6.26 \mathrm{~ns}$ of the syn-enol excitation. Previous experimental studies showed that the lifetime of $\mathrm{HBO}$ was increased from ca. 10 ps at room temperature to $5.7 \mathrm{~ns}$ at $77 \mathrm{~K}$ as the molecular motion was restricted at the $77 \mathrm{~K} .^{[21]}$ The authors also reported that the quantum efficiency of $\mathrm{HBO}$ increased from $3.5 \%$ at room temperature to $37 \%$ at 77 $\mathrm{K}$. These values of the lifetime and the quantum efficiency are in good agreement with what we observed from the DBO solution in THF and DBO-PCDA nanoparticles if we consider the solidstate as a mimic of the frozen solution at $77 \mathrm{~K}$ considering the restricted molecular movement in both cases.

To monitor its photochemical stability, DBO-PCDA nanoparticles were exposed to a 6-W 254-nm handheld UV light held
$1 \mathrm{~cm}$ above the sample for $30 \mathrm{~min}$. The fluorescence intensity of DBO-PCDA nanoparticles decreased only by $15 \%$ upon the intense UV irradiation while other fluorescent organic dyes ${ }^{[1]}$ (Alexa Fluor 488) and conjugated polymers ${ }^{[22]}$ (poly ( $p$-phenyleneethynylene) and poly(3-hexylthiophene)) experienced 80 $90 \%$ of quenching under the same condition (data not shown).

We further investigated the possible application of the highly emissive and biotin-functionalized DBO-PCDA nanoparticles for immunofluorescence labeling. First, sulfo- $N$-hydrosuccinimide-biotin ( $1 \mathrm{mg} \mathrm{ml}^{-1}$ in phosphate buffered saline (PBS)) was spotted on an amine-functionalized glass surface using a manual micro-arrayer (V\&P Scientific, VP478A) (Fig. 3A). Remaining active amines were blocked with poly(ethylene oxide) (see details in the Supporting Information) and subsequently avidin from egg-white $\left(1 \mathrm{mg} \mathrm{ml}^{-1}\right.$ in PBS) was spread on the biotin-patterned glass slide. After thoroughly washing off unbound avidin, a $0.5 \mathrm{mM}$ solution of the self-assembled DBO-PCDA nanoparticles in HEPES buffer was finally applied on the avidin-patterned glass slide. After removing non-specifically bound DBO-PCDA nanoparticles by rinsing several times with HEPES buffer, microscopic images were 


\section{(A)}
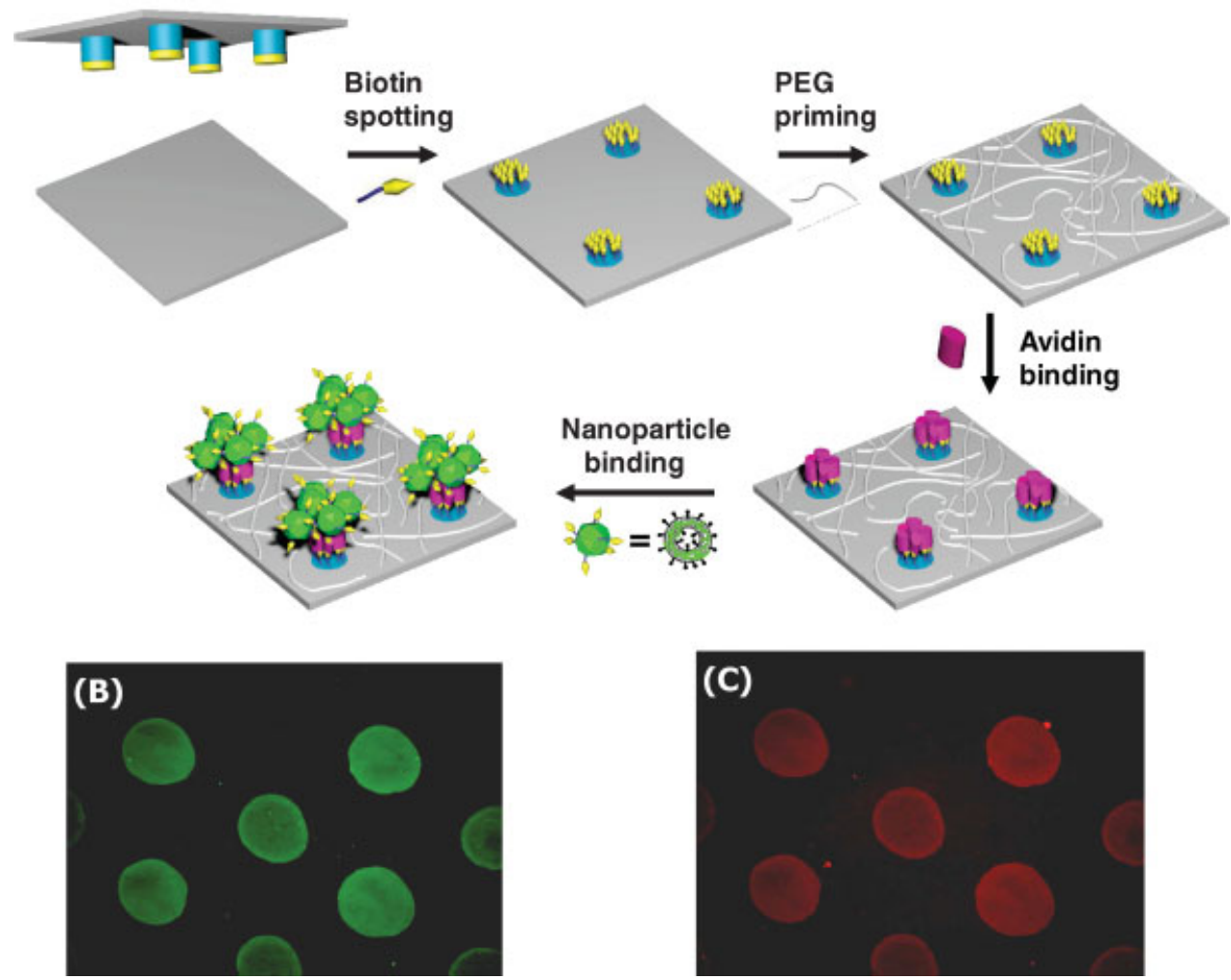

Figure 3. A) Schematic illustration of selective fluorescence labeling by means of functionalized DBO-PCDA organic nanoparticles. B) A fluorescence microscopy image obtained by using a $400 \mathrm{~nm}$ long-pass emission filter after selective binding of biotinylated DBO-PCDA nanoparticles on the patterned avidin surface. C) A fluorescence microscopy image from a $600 \mathrm{~nm}$ long-pass emission filter showing the red fluorescence emission from the red phase of polydiacetylene passivation layer induced by biotin-avidin interaction. The diameter of the dots of the fluorescence microscopy images is $500 \mu \mathrm{m}$.

taken as shown in Figure 3B. As we can see from the image green fluorescent was observed from only the avidin spots, demonstrating good selectivity and bright emission.

The self-assembled DBO-PCDA also has a dual color capability because the PCDA not only serves as a surfactant and a recognition unit for the target avidin spots but also can generate a sensory signal. We polymerized the PCDA passivation layer after self-assembling the biotinylated DBO-PCDA nanoparticles. The polymerized polydiacetylene (PDA) layer will experience perturbation of the conjugated backbone and undergo mechanochromism from the blue form to the red form during the biotin-avidin recognition/binding. Because the red form is emissive ${ }^{[23]}$ as we anticipated we observed red emission when we used a $600 \mathrm{~nm}$ long-pass emission filter with a $550 \mathrm{~nm}$ excitation filter as shown in Figure 3C.

In summary, we have synthesized DBO and self-assembled DBO with functionalized PCDA molecules to form welldefined DBO-PCDA nanoparticles. The DBO-PCDA nanoparticles showed aggregation-induced fluorescence enhancement having a $38 \%$ of quantum yield. Intermolecular hydrogen bonding is believed to form J-aggregates and allows intermolecular excited state proton transfer. Selective targeting and dual color visualization of patterned avidin arrays were achieved by using the highly emissive biotinylated DBO-PCDA nanoparticles to demonstrate the promising application of the organic nanoparticles for immunofluorescence labeling.

\section{Experimental}

Glass slides were cleaned in boiling $\mathrm{H}_{2} \mathrm{O}: \mathrm{H}_{2} \mathrm{O}_{2}: \mathrm{NH}_{4} \mathrm{OH}$ (1:1:4 volume ratio) solution for $30 \mathrm{~min}$, followed by piranha solution $\left(\mathrm{H}_{2} \mathrm{O}_{2}: \mathrm{H}_{2} \mathrm{SO}_{4}\right.$ 3:7) etching after thorough rinsing with water and drying. The glass slides were further dipped in $2 \mathrm{wt} \% 3$-aminopropyltriethoxysilane in toluene solution for $1 \mathrm{~h}$, and were sonicated in toluene: methanol (1:1) and methanol for $3 \mathrm{~min}$ each to remove unbound silanes. The final amine functionalized glass slides were stored in a sealed container at $4^{\circ} \mathrm{C} .1 \mathrm{mg}$ of sulfo-NHS-biotin purchased from Aldrich was dissolved in $1 \mathrm{ml}$ of phosphate buffered saline and used right after the preparation before the hydrolysis of sulfo-NHS. $2.2 \mathrm{mg}$ succinimidyl-4-( $N$-maleimidomethyl)cyclohexane-1-carboxylate (purchased from Pierce, Co.) was dissolved in $200 \mu \mathrm{l}$ DMSO and further diluted in DMSO:ethanol $2: 8$ volume ratio mixture. $2.4 \mathrm{mg}$ of mono functional thiolated poly(ethylene oxide) $\left(M_{\mathrm{w}}=2400 \mathrm{~g} \mathrm{~mol}^{-1}\right.$, purchased from PolymerScience) in $10 \mathrm{ml}$ of PBS. $1 \mathrm{mg}$ of Avidin from egg-white purchased from Aldrich was also dissolved in $1 \mathrm{ml}$ of PBS buffer with $0.02 \mathrm{wt} \%$ Tween20 (PBST). Sulfo-NHS-biotin was spotted on an amine glass with a manual microarray (V\&P Scientitic, VP475A) and stored in a humidity chamber (Relative humidity $\sim 90 \%$ ) for $4 \mathrm{~h}$. After rinse with PBS buffer, remaining active amines were reacted with SMCC crosslinker and were blocked further with thiol-poly(ethylene oxide) in PBS and rinsed with water. Avidin solution was spread on the biotin spotted glass 
for $30 \mathrm{~min}$. After the avidin-biotin binding, the unbound avidin was rinsed off with PBST and stored in PBS until next experiment. The avidin patterned glass slide was immersed in the biotinylated vesicle suspension for $30 \mathrm{~min}$ and examined with a fluorescence microscope (Olympus BX51) $3.5 \mu \mathrm{mol}$ of PCDA-ABA and $1.5 \mu \mathrm{mol}$ of PCDABiotin were dissolved in chloroform and filtered through a $0.45 \mu \mathrm{m}$ syringe filter. After the evaporation of chloroform, $10 \mathrm{ml}$ of $1 \mathrm{mM}$ HEPES buffer was added in to give $0.5 \mathrm{mM}$ PCDA. The homogeneous PCDA suspension was obtained via sonication for $15 \mathrm{~min}$ at the transition temperature of PCDA. Pre-determined amount $(0.1 \mathrm{ml})$ of $1 \mathrm{mM}$ DBO in THF solution was injected after or during the vesicle formation to get 1:50 DBO:PCDA mol ratio. And the final DBOPCDA vesicles were stored at $4{ }^{\circ} \mathrm{C}$ in the dark before use. Nanoparticles in THF: $\mathrm{H}_{2} \mathrm{O} 1: 9$ mixture were obtained by precipitation of $1 \mathrm{ml}$ of 0.1 $\mathrm{mM}$ DBO in THF into $9 \mathrm{ml}$ of deionized water during sonication.

Received: July 04, 2007 Revised: September 27, 2007 Published online: February 29, 2008

[1] X. Wu, H. Liu, J. Liu, K. N. Haley, J. A. Treadway, J. P. Larson, N. Ge, F. Peale, M. P. Bruchez, Nat. Biotechnol. 2003, 21, 41.

[2] M. Bruchez, Jr, M. Moronne, P. Gin, S. Weiss, A. P. Alivisatos, Science 1998, 281, 2013.

[3] W. C. Chan, S. Nile, Science 1998, 281, 2016.

[4] X. Michalet, F. F. Pinaud, L. A. Bentolila, J. M. Tsay, S. Doose, J. J. Li, G. Sundaresan, A. M. Wu, S. S. Gambhir, S. Weiss, Science 2005, 307, 538.

[5] M. A. Hines, P. Guyot-Sionnest, J. Phys. Chem. 1996, 100, 468.

[6] C. J. Murphy, Anal. Chem. 2002, 74, 520A.
[7] W. J. Parak, D. Gerion, T. Pellegrino, D. Zanchet, C. Micheel, S. C. Williams, R. Boudreau, M. A. Le Gros, C. A. Larabell, A. P. Alivisatos, Nanotechnology 2003, 14, R15.

[8] C. M. Niemeyer, Angew. Chem. Int. Ed. 2001, 40, 4128

[9] P. Alivisatos, Nat. Biotechnol. 2004, 22, 47.

[10] A. M. Derfus, W. C. W. Chan, S. N. Bhatia, Nano Lett. 2004, 4, 11.

[11] C. Kirchner, T. Liedl, S. Kudera, T. Pellegrino, A. M. Javier, H. E. Gaub, S. Stölzle, N. Fertig, W. J. P. Parak, Nano Lett. 2005, 5, 331.

[12] B.-K. An, D.-S. Lee, J.-S. Lee, Y.-S. Park, H.-S. Song, S. Y. Park, J. Am. Chem. Soc. 2004, 126, 10232.

[13] S. Kim, Q. Zheng, G. S. He, D. J. Bharali, H. E. Pudavar, A. Baev, P. N Prasad, Adv. Funct. Mater. 2006, 16, 2317.

[14] S. Kim, T. Y. Ohulchanskyy, H. E. Pudavar, R. K. Pandey, P. N. Prasad J. Am. Chem. Soc. 2007, 129, 2669.

[15] Y. Li, F. Li, H. Zhang, Z. Xie, W. Xie, H. Xu, B. Li, F. Shen, L. Ye, M. Hanif, D. Ma, Y. Ma, Chem. Commun. 2007, 231.

[16] Z. Li, Y. Dong, B. Mi, Y. Tang, M. Häussler, H. Tong, Y. Dong, J. W. Y. Lam, Y. Ren, H. H.-Y. Sung, K. S. Wong, P. Gao, I. D. Williams, H. S. Kwok, B. Z. Tang, J. Phys. Chem. B 2005, 109, 10061.

[17] G. L. Silva, V.Ediz, D. Yaron, B. A. Armitage, J. Am. Chem. Soc. 2007, 129,5710

[18] D. Horn, J. Rieger, Angew. Chem. Int. Ed. 2001, 40, 4330.

[19] Supporting Information of synthesis of PCDA: J.-M. Kim, J.-S. Lee, H. Choi, D. Sohn, D. J. Ahn, Macromolecules 2005, 38, 9366.

[20] O. K. About-Zied, R. Jimenez, E. H. Z. Thompson, D. P. Millar, F. E. Romesberg, J. Phys. Chem. A 2002, 106, 3665.

[21] K. Das, N. Sarkar, A. Kumar, G. D. Majumdar, D. N. Nath, K Bhattacharyya, J. Phys. Chem. 1994, 98, 9126.

[22] K. Lee, H.-J. Kim, J. C. Cho, J. Kim, Macromolecules 2007, 40, 6457.

[23] J. Olmsted, III, M. Strand, J. Phys. Chem. 1983, 87, 4790. 